\title{
Ultracompact resonant filters in photonic crystals
}

\author{
T. P. White \\ Centre for Ultrahigh-Bandwidth Devices for Optical Systems and School of Physics, University of Sydney, Sydney, NSW 2006, Australia
}

\section{C. Botten}

Centre for Ultrahigh-Bandwidth Devices for Optical Systems and School of Mathematical Sciences, University of Technology, Sydney, P.O. Box 123, Broadway, NSW 2007, Australia

\section{R. C. McPhedran and C. Martijn de Sterke}

Centre for Ultrahigh-Bandwidth Devices for Optical Systems and School of Physics, University of Sydney, Sydney, NSW 2006, Australia

Received June 30,2003

A novel design for an ultracompact, high- $Q$ notch-rejection filter is presented, and an analytic expression for the transmission properties is derived. This folded directional coupler shares the properties of a Fabry-Perot resonator and a directional coupler. We compare and contrast the device to high- $Q$ Fabry-Perot cavities in photonic crystal waveguides. (C) 2003 Optical Society of America

OCIS codes: $\quad 050.2230,130.1750,230.5750,230.7370$.

Optical integrated circuits, or photonic chips, are expected to be critical components of future high-capacity optical communications systems. These multifunction devices will perform signal processing tasks that are currently carried out electronically. The main advantages of transferring to all-optical technology are the superior bandwidth at optical frequencies and the corresponding increase in data transfer rate that it provides. A necessary requirement for photonic chips is that they have a small size, which places strict limits on the sizes of individual components, and many novel devices have been designed and demonstrated with the aim of compactness. Such components range in length from tens of micrometers for fused vertical directional couplers, ${ }^{1}$ for which two waveguides are fused one on top of the other, to high-transmission $90^{\circ}$ bends on a scale of a few micrometers. ${ }^{2}$ Directional couplers with coupling lengths of several wavelengths have also been demonstrated in photonic crystals. . $^{3,4}$

Here we introduce the folded directional coupler (FDC): a novel, ultracompact, high- $Q$ notch-rejection filter that exploits the mode-coupling properties of a directional coupler and the sharp resonances of a Fabry-Perot (FP) resonator. Filters are essential components in optical networks, for which channel rejection and channel passing filters may both be required. As network capacity increases and channel spacing is reduced in wave-division multiplexing systems, the spectral responses of such filters must become narrower. Figure 1 shows the general structure of a FDC in a photonic crystal, which consists of a single-mode input waveguide (region M1), a single-mode output waveguide (M3), and an overlap region (M2) of length $L$ where the modes of the two guides couple. A simple analytic model based on mode coupling and reflection at the ends of region M2 describes the transmission properties of the FDC accurately, and we compare the results with those from full numerical calculations. We show that an ideal FDC structure that is less than $3 \mu \mathrm{m}$ long can be tuned to have a notch-rejection width of less than $10 \mathrm{GHz}$, corresponding to a quality factor $Q=\omega_{0} /\left(2 \Delta \omega_{1 / 2}\right)=$ 22,000 , where $\omega_{0}$ is the central frequency of the response and $2 \Delta \omega_{1 / 2}$ is the FWHM of the response peak.

The concept of combining FP effects in a directional coupler geometry was discussed by Safaai-Jazi and Chang ${ }^{5}$ as a way to improve mode discrimination and longitudinal mode spacing in resonant cavities. Although the FDC shares similarities with this device, there are also distinct differences. First, the FDC is a two-port device, with only one input and one output waveguide. Second, at each end of the interaction region in the FDC, one of the waveguides is blocked by a fully reflective element and the other is open, whereas the device discussed in Ref. 5 has a partially reflecting mirror at each end of the cavity, covering both guides.

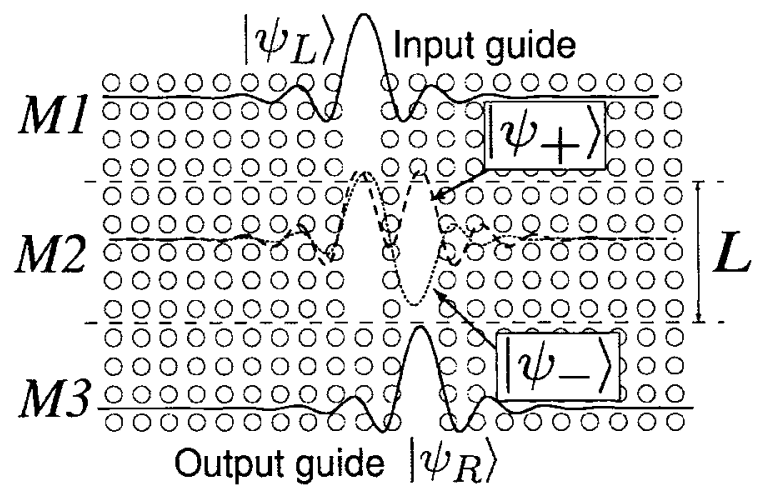

Fig. 1. FDC with guide overlap region M2 of length $L=$ $5 d$ and a single line of cylinders separating the two guides. The odd and even supermodes of region M2, $\left|\psi_{ \pm}\right\rangle$, are indicated by the dashed and dotted curves, respectively. The left and right modes, $\left|\psi_{L, R}\right\rangle$, of the single-guide regions are shown as solid curves.

(C) 2003 Optical Society of America 
This difference in the reflection mechanism allows the FDC to exhibit a transmission behavior that is complementary to that of FP devices. FP structures are normally highly reflective, transmitting only when a resonance condition is satisfied (notch-pass filter) but the FDC normally transmits, and it reflects only at resonance (notch-rejection filter). We discuss a design for a photonic crystal FP filter with performance parameters similar to those of the FDC described later in this Letter.

The resonances of the FDC are accurately described by a simple analytic model that requires only the modal propagation constants in region $\mathrm{M} 2$, and the length of the overlap regions, as inputs. The propagation constants are calculated by use of a full numerical treatment. ${ }^{6}$ This model not only gives an insight into the underlying physics of the FDC but also provides a powerful and efficient tool for the optimization of designs. The derivation of the model relies on expressing the propagating mode fields on two different bases. The first mode basis consists of the fundamental modes of the input (left) and output (right) guides, denoted $\left|\psi_{L}\right\rangle$ and $\left|\psi_{R}\right\rangle$, respectively, as illustrated in Fig. 1. In the overlap region the two single modes of the individual guides form symmetric (even) and antisymmetric (odd) supermodes of the double waveguide, $\left|\psi_{+}\right\rangle$ and $\left|\psi_{-}\right\rangle$, respectively. The propagation constants of these supermodes are labeled $\beta_{+}$and $\beta_{-}$, and we define $\Delta \beta=\left|\beta_{+}-\beta_{-}\right| / 2$ and $\bar{\beta}=\left(\beta_{+}+\beta_{-}\right) / 2$; so the beat length is $L_{B}=\pi / \Delta \beta$.

If the single guide modes are not distorted significantly when the guides are brought close together, the modes in the two bases are related by

$$
\left|\psi_{L}\right\rangle=\left(\left|\psi_{+}\right\rangle+\left|\psi_{-}\right\rangle\right) / \sqrt{2}, \quad\left|\psi_{R}\right\rangle=\left(\left|\psi_{+}\right\rangle-\left|\psi_{-}\right\rangle\right) / \sqrt{2} .
$$

Light incident upon the overlap region from the input guide in mode $\left|\psi_{L}\right\rangle$ is expressed in terms of $\left|\psi_{+}\right\rangle$and $\left|\psi_{-}\right\rangle$by Eqs. (1). The even and odd supermodes then propagate through $\mathrm{M} 2$ with different propagation constants, advancing in phase by factors $\exp \left(i \beta_{+} L\right)$ and $\exp \left(i \beta_{-} L\right)$, respectively. At the interface with $\mathrm{M} 3$ the fields are reexpressed in terms of $\left|\psi_{L}\right\rangle$ and $\left|\psi_{R}\right\rangle$; in this simple model the component of the field in mode $\left|\psi_{R}\right\rangle$ is taken to be transmitted into the output guide, and the field in mode $\left|\psi_{L}\right\rangle$ to be reflected without a phase change. The reflected field is then transformed into a \pm basis by use of Eqs. (1) and propagated back through region M2 to the interface with M1. Again, the field is separated into left and right components. The left component propagates into the input guide, and the right component is reflected back into M2. Successive reflections into M1 from each pass through M2 add coherently to give a geometric series, leading to reflection coefficient $\rho$ and reflectivity $R=|\rho|^{2}$ :

$$
R=\frac{\cos ^{4}(\Delta \beta L)}{\cos ^{4}(\Delta \beta L)+4 \sin ^{2}(\Delta \beta L) \cos ^{2}(\bar{\beta} L)}
$$

When $L=L_{B}$ in Eq. (2), $R=1$ as expected, as the incident field returns to the left guide of the FDC at the end of region M2 and is reflected directly back into the input guide. Similarly, when $L=L_{B} / 2$ the incident field crosses over into the right guide at the end of M2 and is transmitted into the output guide, and thus $T=1$. For the purpose of designing a notch-rejection filter, we are interested in the resonance conditions for which $R=1$. Examining Eq. (2), one can see that in addition to the case mentioned above, these conditions occur when $\bar{\beta} L=\pi / 2,3 \pi / 2,5 \pi / 2 \ldots$ This is the resonance condition used for the filter.

Suppose that the resonant frequency is $\omega=\omega_{0}$ and denote the values of $\Delta \beta$ and $\bar{\beta}$ at resonance by $\Delta \beta_{0}$ and $\bar{\beta}_{0}$. We approximate the shape of the resonance peak at $\omega=\omega_{0}+\Delta \omega$ by expanding $\Delta \beta$ and $\bar{\beta}$ to first order; thus $\Delta \beta=\Delta \beta_{0}+\Delta \omega \Delta \beta_{1}$ and $\bar{\beta}=\bar{\beta}_{0}+\Delta \omega \bar{\beta}_{1}$, where $\Delta \beta_{1}=(\mathrm{d} \Delta \beta / \mathrm{d} \omega)_{\left(\omega=\omega_{0}\right)}$, and $\bar{\beta}_{1}$ is similarly defined. Substituting these expansions into Eq. (2) and expanding to first order, we obtain

$$
\rho=\frac{-1}{1-i\left(\Delta \omega / \Delta \omega_{1 / 2}\right)}, \quad R=\frac{1}{1+\left(\Delta \omega / \Delta \omega_{1 / 2}\right)^{2}},
$$

where $R=1 / 2$ at $\omega=\omega_{0} \pm \Delta \omega_{1 / 2}$ and

$$
\Delta \omega_{1 / 2}=\cos ^{2}\left(\Delta \beta_{0} L\right) /\left(2 \bar{\beta}_{1} L\right) .
$$

Equations (2)-(4) show that the position of the resonance for a structure of length $L$ is largely determined by $\bar{\beta}$, whereas the peak width $\left(2 \Delta \omega_{1 / 2}\right)$ is determined by $\Delta \beta$. Numerical studies indicate that $\bar{\beta}$ varies only slightly from the propagation constant of a single isolated guide, even when the two guides in region M2 are close together, whereas $\Delta \beta$ is a much stronger function of the structural parameters in M2. One thus can tune the peak half-width given by Eq. (4) by adjusting the structure of region $\mathrm{M} 2$, and hence $\Delta \beta$, for instance, by changing the separation of the guides or by modifying the separating cylinders. We discuss tuning of the FDC further below. In contrast, the $Q$ of a FP resonator of fixed length, operating at a fixed wavelength, depends only on the mirrors' reflectivity, $|r|^{2}$; the peak width ${ }^{7}$ is proportional to $\left(1-|r|^{2}\right) /|r|$. Thus high- $Q$ FP cavities are possible only with highly reflective mirrors.

To validate the approximations that we used to derive Eqs. (2)-(4) we compared our full numerical results for the FDC. We found that, for best agreement, parameter $L$ in the model must be varied somewhat from length $L$ defined in Fig. 1 . This variation accounts for the phase change on reflection of the mode in the blocked guide. The numerical results were calculated by a new method based on the propagation of Bloch modes within each distinct section of waveguide and on the scattering of these modes at interfaces with other waveguides. ${ }^{6}$ This rigorous treatment includes all evanescent modes in the structure, but, even when only the propagating modes are considered for the FDC structure considered here, the agreement with the full calculation is excellent. A comparison of the analytic form [Eq. (3)] and the full numerical calculation is shown in Fig. 2, where the transmissivity, $T=1-R$, is plotted near resonance and shows this good agreement. The spectrum is for the FDC structure illustrated in Fig. 1, formed in a square photonic crystal lattice 


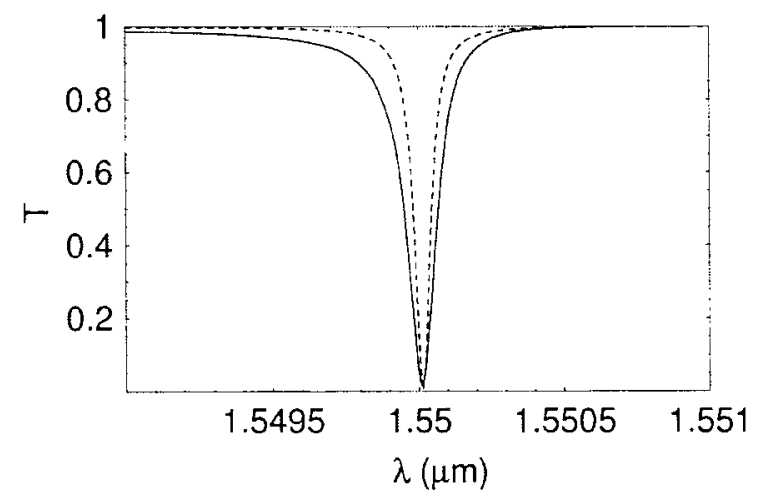

Fig. 2. Transmission of the FDC in Fig. 1 near resonance at $\lambda=1.55 \mu \mathrm{m}$. Solid curve, full numerical calculation; dashed curve, approximate result obtained from Eqs. (3) with numerical input of $\bar{\beta}, \Delta \beta$, and $L$.

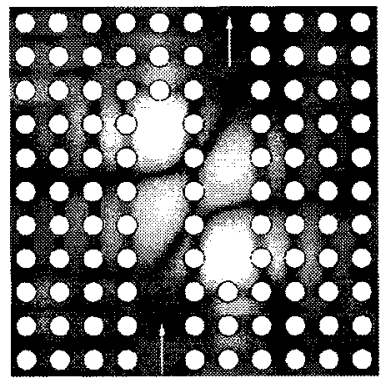

(a)

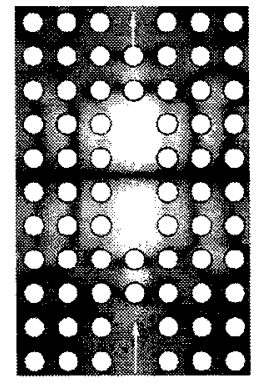

(b)
Fig. 3. (a) Field intensity in the FDC at resonance. The intensity peaks at the blocked end of each waveguide correspond to an intensity enhancement of more than 4 orders of magnitude. (b) Field intensity of a photonic crystal FP filter with cavity length $L=4$. Shading in both figures is on a logarithmic scale.

(period $d$ ) of dielectric cylinders with radius $a=0.3 d$ and refractive index $n=3.0$ in air. This photonic crystal has a bandgap for TM-polarized light in the wavelength range $2.9 d<\lambda<3.8 d$. All calculations presented here are for wavelengths within this range. The cylinder separation of $d=0.513 \mu \mathrm{m}$ was chosen to give a resonance peak at $\lambda=1.55 \mu \mathrm{m}$. For these parameters the length of the overlap region is only $L=5 d=2.6 \mu \mathrm{m}$, or 1.7 wavelengths, and $Q>22,000$, giving a peak width of $8 \mathrm{GHz}$, or $\Delta \lambda=0.07 \mathrm{~nm}$. This resonance results in a strong-field enhancement in region $M 2$. The field intensity in the $F D C$ at $\lambda=1.55 \mu \mathrm{m}$ is shown in Fig. 3(a), where the peak intensity is more than 4 orders of magnitude greater than the input intensity.

The parameters for the device described above are not optimized and were chosen to demonstrate the short length and high $Q$ that are possible in a FDC with no tuning of the interaction region. By adjusting the radius of the cylinders separating the two waveguides in region $\mathrm{M} 2$ to $a=0.29 d$ we can design a FDC with length $L=4 d=2 \mu \mathrm{m}$ and $Q=3600$, corresponding to $\rfloor \omega=54 \mathrm{GHz}$, or $\Delta \lambda=0.4 \mathrm{~nm}$.

For comparison with the FDC, we studied a simple FP filter in a photonic crystal waveguide consisting of a single-mode waveguide in the same photonic crystal as the FDC examples above. We formed reflective interfaces by blocking the waveguide with two plugs of cylinders, separated by a length $L$, to create a resonant cavity. The reflectivity of the plugs, and hence the $Q$ of the cavity, can be increased by stacking more cylinders, effectively placing a slab of photonic crystal in the waveguide. The resonant field intensity in a FP device of this type, with length four cylinders and plugs two cylinders deep, is shown in Fig. 3(b). For cylinders of $a=0.3 d$, as in the bulk crystal, we found that the plug must be more than three cylinders deep to achieve $|r|^{2}=0.998$, necessary for a $Q$ value of 3600 in a FP structure with a cavity of length $5 d$. Including the mirrors, the total length of this device is $11 d$, which is more than $5 \mu \mathrm{m}$ at $\lambda=1.55 \mu \mathrm{m}$. Although the FP filter behaves as a notch-pass rather than as a notch-rejection filter, the comparison with the FDC is interesting. By stacking more layers of cylinders in the plug one can increase the $Q$ of the FP filter at the expense of creating a longer device, whereas we can narrow the response of the FDC by tuning the interaction region at a fixed length. Thus the minimum length of the FDC is restricted only by the geometry and by evanescent mode coupling across the cavity.

In conclusion, we have demonstrated a new class of notch-rejection filter that exploits the sharp spectral response of a Fabry-Perot resonator and the mode-coupling behavior of a directional coupler. This geometry allows ultrashort, high- $Q$ filters to be designed in photonic crystals, with potential applications in optical integrated circuits and optical networking components. A simple mode-coupling model accurately describes the transmission behavior of the device close to the resonant frequency. Although photonic crystals provide a number of natural advantages for FDC fabrication, such as total reflection at the ends of the blocked guides, the behavior is not limited to photonic crystals. FDC structures could also be formed in dielectric waveguides with highly reflective mirrors at the guide ends.

Support of the Center of Excellence for UltrahighBandwidth Devices for Optical Systems by the Australian Research Council is acknowledged. T. P. White's e-mail address is twhite@physics.usyd.edu.au.

\section{References}

1. S. Cho, B. Kim, Y. Moon, and A. Shakouri, Jpn. J. Appl. Phys. 40, 4045 (2001).

2. A. Mekis, J. C. Chen, I. Kurland, S. Fan, P. Villeneuve, and J. D. Joannopoulos, Phys. Rev. Lett. 77, 3787 (1996).

3. A. Martinez, F. Cuesta, and J. Marti, IEEE Photon Technol. Lett. 15, 694 (2003).

4. M. Koshiba, J. Lightwave Technol. 19, 1970 (2001).

5. A. Safaai-Jazi and C. Chang, IEEE J. Quantum Electron. 32, 1063 (1996).

6. L. C. Botten, A. A. Asatryan, T. N. Langtry, T. P. White, C. M. de Sterke, and R. C. McPhedran, Opt. Lett. 28, 854 (2003).

7. M. Born and E. Wolf, Principles of Optics, 5th ed. (Pergamon, Oxford, 1975), Sec. 7.6.1. 